\title{
Speed of Propagation for Some Models of Two-Phase Flow in Porous Media
}

\author{
C. E. Kees \\ Center for Research in Scientific Computation, Department of Mathematics, North Carolina State University, \\ Raleigh, North Carolina, USA
}

\begin{abstract}
Flow of two immiscible, incompressible fluids in a porous medium is typically described by a nonlinear advection-diffusion equation for one of the fluid saturations. The diffusion coefficient, which represents the effect of capillary forces on the fluids, is zero when the medium is locally saturated by either fluid since in these limiting cases the effects of capillary forces tend to zero. This degeneracy in the second-order term usually gives rise to the qualitative property that perturbations in saturation propagate with finite speed through regions that are fully saturated by either fluid. This qualitative property is physically realistic. In this work we show that, under certain choices of constitutive relations and modeling approximations, the finite speed of propagation property is lost, despite the fact that the diffusion coefficient is degenerate. The loss of finite speed of propagation is due to unbounded derivatives in the closure relations as the medium becomes saturated by wetting phase. We present analytical and numerical solutions, compare solution dynamics that display finite and infinite speed of propagation, and provide a brief account of numerical difficulties related to the degenerate coefficients.
\end{abstract}

\section{Introduction}

Models of two-phase flow in porous media are fundamental tools for understanding subsurface water resources. They describe soil moisture in the unsaturated zone as well as transport of industrial solvents in aquifers. Models of twophase flow must mimic static and dynamic conditions in two-phase systems to be useful, whether they are used to make predictions, to design remediation strategies, or simply to provide physical intuition. Nevertheless, in representing nature as a mathematical model, some natural behavior is lost and some unnatural behavior is gained. In this respect, good models present acceptable trade-offs between physical and non-physical behavior. This technical note focuses on the way perturbations propagate in solutions of certain two-phase model equations and the consequences of this behavior on numerical methods. The more important question of whether the solution behavior is an acceptable approximation of nature would need to be addressed by experiments or deeper theoretical results.

We consider models based on continuum mass balance equations and the multi-phase extension of Darcy's law, derived, for example, using the representative elementary volume approach [Bear, 1972]. The fluids are assumed to be incompressible, and the media is assumed to be incompressible and immobile. This approach results in two conservation laws relating the saturations of the fluids to the fluid pressure gradients and to the gravitational forces via nonlinear proportionality constants called the relative permeabilities. Mathematical closure is obtained by following the approach outlined in Parker et al. [1987], which uses the capillary pressure model described in van Genuchten [1980] and the permeability model described in Mualem [1976]. The result is a coupled system of second-order quasilinear equations that are of degenerate parabolic type. That is, except at two discrete values of saturation, the diffusion coefficients are strictly positive, and all of the nonlinear coefficients are at least once differentiable. In one dimension the system of equations can be reduced further to a single degenerate parabolic equation. This work focuses strictly on the behavior of the one-dimensional model equation.
The qualitative behavior of solutions of partial differential equations is particularly important in the case of spatially variable or nonlinear coefficients, where analytical solutions are not generally available. When something is known about the qualitative behavior of solutions, such as a maximum principle or conservation of mass, these properties can be recovered in a discrete sense by carefully designing numerical methods. Gilding and Kersner [1996] gives a very complete classification of scalar nonlinear advection-diffusion-reaction equations in terms of finite speed of propagation, which is a qualitative property of solutions.

To define the finite speed of propagation property we begin by considering non-negative functions $u(x, t)$ (i.e. solutions of partial differential equations) which are defined on a half strip $(0, \infty) \times(0, T]$. We define the support of $u$ as

$$
P[t]=\{x \in(0, \infty) \mid u(x, t)>0\}
$$

and the front or free boundary as

$$
\zeta(t)=\sup _{x \in(0, \infty)} P[t]
$$

We say that $u$ has the finite speed of propagation property if

$$
\zeta(t)<\infty \quad t \in[0, \tau)
$$

for some $\tau>0$. That is, if the support of $u$ is bounded initially then it stays bounded over finite time intervals. The property is related to the existence of finite or bounded traveling wave solutions of the partial differential equations, as is shown in Gilding and Kersner [1996]. If $u$ is some measure of the amount of a substance at location $x$ at time $t$, and that substance is merely transported by natural processes, then it seems reasonable to require that solutions of the model equation not cause the substance to occupy an unbounded region in finite time. Equivalently, we expect that $\zeta(t)$ is always finite. If a column of porous media is initially saturated with fluid $W$ and another fluid $N$ is injected at the bottom of the column, then the finite speed of propagation implies that $N$ will not flow out of the top of the column instantaneously. 
The linear advection-diffusion equation does not possess the finite speed of propagation property: solutions with bounded initial support are instantaneously positive on the entire half line. This behavior in the mathematical model is acceptable in most cases because the solution decays rapidly enough that it provides a good approximation of nature. Nonlinear hyperbolic partial equations usually have finite speed of propagation. On the other hand, nonlinear hyperbolic equations have discontinuous solutions, which, although non-physical in many applications, are often good approximations at the scale of observation. The objective of this work is to demonstrate a non-physical aspect of solutions of the model equation, which may or may not be an acceptable approximation to nature, and to examine how this aspect affects numerical methods.

In section 2 we present the two-phase flow model and Richards' equation for air/water systems. We also derive some asymptotic approximations to the nonlinear parameters that will be used to formulate simplified model equations and to analyze the speed of propagation. We apply the results of Gilding and Kersner [1996] to the two-phase models in section 4 and identify the range of parameters that lead to infinite speed of propagation. In section 4 we present analytical solutions of the equations for several special cases in order to contrast the behavior of solutions with and without finite speed of propagation. Finally, in section 5, we describe some simple numerical approaches for solving the equations and demonstrate additional consequences of the degenerate form of the equations.

\section{Two-Phase Flow Models}

In one space dimension, the standard model for incompressible two-phase flow in a homogeneous porous medium reduces to an equation for the wetting phase saturation $s$ (c.f. [Chavent and Jaffré, 1986]):

$$
s_{t}+\left[f(s)-d(s) s_{x}\right]_{x}=0
$$

where

$$
\begin{aligned}
d= & -\frac{k_{i}}{\omega \mu_{w}} \frac{k_{r w} k_{r n}}{\frac{\mu_{n}}{\mu_{w}} k_{r w}+k_{r n}} \frac{d p_{c}}{d s} \\
f= & \frac{k_{i} \mu_{n}}{\omega \mu_{w}} \frac{k_{r w}}{\frac{\mu_{n}}{\mu_{w}} k_{r w}+k_{r n}} q_{t} \\
& +\frac{k_{i}}{\omega \mu_{w}} \frac{k_{r w} k_{r n}}{\frac{\mu_{n}}{\mu_{w}} k_{r w}+k_{r n}}\left(1-\frac{\rho_{n}}{\rho_{w}}\right) \rho_{w} g_{x}
\end{aligned}
$$

and where $k_{i}$ is intrinsic permeability, $\omega$ is porosity, $\mu_{w}$ is wetting phase viscosity, $\mu_{n}$ is non-wetting phase viscosity, $\rho_{w}$ is wetting phase density, $\rho_{n}$ is non-wetting phase density, $q_{t}$ is total fluid velocity, and $g_{x}$ is gravitational acceleration. The nonlinear constitutive relations are the relative permeabilities, $k_{r w}$ and $k_{r n}$, and the capillary pressure $p_{c}$, which we will specify after considering a related two-phase model. We will refer to $d$ as the diffusion coefficient and $f$ as the advective flux.

\subsection{Richards' Equation for Air/Water Systems}

Our intention in this section is not to derive Richards' equation rigorously but rather to clarify how commonly used forms of Richards' equation relate to the two-phase model above. The original derivation of Richards' equation was based on physical principles rather than as an approximation to the two-phase flow model [Richards, 1931]. Starting from the two-phase flow equation, if we assume $\rho_{n} / \rho_{w}=0$ and $\mu_{n} / \mu_{w}=0$ we obtain

$$
\begin{aligned}
f & =\frac{k_{i} k_{r w}}{\omega \mu_{w}} \rho_{w} g_{x} \\
d & =-\frac{k_{i} k_{r w}}{\omega \mu_{w}} \frac{d p_{c}}{d s}
\end{aligned}
$$

The resulting equation is the saturation form of Richards' equation. Since the density and viscosity of air are in fact much smaller than those of water, the assumption that $\rho_{n} / \rho_{w}=0$ and $\mu_{n} / \mu_{w}=0$ seems like a reasonable approximation.

\subsection{Effective Saturation}

Most closure relations for two-phase flow are based on a reduced or effective saturation defined by

$$
s_{e}=\frac{s-s_{r}}{s_{s}-s_{r}}
$$

where $s_{r}$ is the residual saturation and $s_{s}$ is the maximum wetting phase saturation. This reflects the fact that, if only flow processes are involved, it is usually impossible for either of the two fluids to be completely removed from the medium because of entrapment mechanisms. For this work we are interested in what happens when $s=s_{s}$. For simplicity, we take $s_{s}=1$ and $s_{r}=0$ so that $s_{e}=s$.

\subsection{Relative Permeability}

We will use the wetting phase relative permeability model derived in Mualem [1976], which was later extended to the non-wetting phase in Parker et al. [1987]:

$$
\begin{aligned}
& k_{r w}=s_{e}^{1 / 2}\left\{\frac{\int_{0}^{s_{e}} \frac{1}{p_{c}} d s_{e}}{\int_{0}^{1} \frac{1}{p_{c}} d s_{e}}\right\}^{2} \\
& k_{r n}=\left(1-s_{e}\right)^{1 / 2}\left\{\frac{\int_{s_{e}}^{1} \frac{1}{p_{c}} d s_{e}}{\int_{0}^{1} \frac{1}{p_{c}} d s_{e}}\right\}^{2}
\end{aligned}
$$

The analytical form of these functions depends on the capillary pressure.

\subsection{Capillary Pressure}

The capillary pressure is expressed as a function of saturation according to the model presented in van Genuchten [1980]:

$$
p_{c}=\frac{1}{\alpha}\left(s_{e}^{-1 / m}-1\right)^{1 / n}
$$

where $\alpha, n$, and $m$ are constants.

\subsection{Van Genuchten-Mualem Closure Relations}

Following [van Genuchten, 1980], we require that $m=$ $1-1 / n$, so that the integral in the permeability relations above can be integrated exactly. The resulting closure relations can be written as

$$
\begin{aligned}
\frac{d p_{c}}{d s_{e}} & =\frac{m-1}{m \alpha}\left(s_{e}^{-1 / m}-1\right)^{-m}\left(s_{e}^{-1 / m-1}\right) \\
k_{r w} & =s_{e}^{1 / 2}\left[1-\left(1-s_{e}^{1 / m}\right)^{m}\right]^{2} \\
k_{r n} & =\left(1-s_{e}\right)^{1 / 2}\left(1-s_{e}^{1 / m}\right)^{2 m}
\end{aligned}
$$

for $s_{e} \in[0,1]$ where $0<m<1$ and $\alpha>0$ are constants.

For notational simplicity we will set $\alpha=\omega=k_{i}=\rho_{w}=$ $\mu_{w}=\mu_{n}=1$, and $\rho_{n}=0$. Additionally, we consider only cases with $q_{t}=0$ and $g_{x}=-1$ or 0 . The case with $g_{x}=-1$ is called gravity segregation or counter-current flow, since 


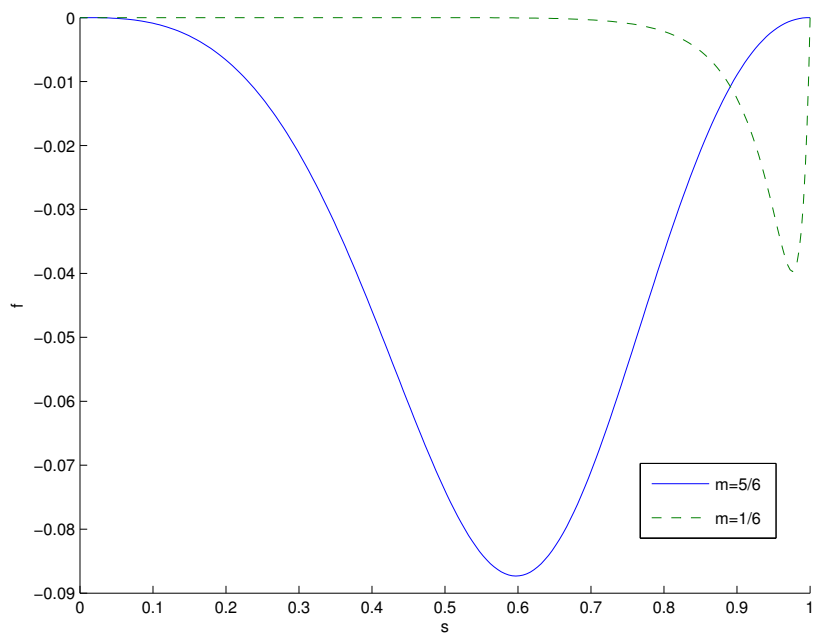

Figure 1. Advective flux, $f$, versus saturation, $s$, for two-phase flow. For all $m, f(0)=f(1)=0$. For small $m, \frac{d f}{d s}(1)=\infty$. Consequently, $f$ is not Lipschitz continuous at $s=1$ for small $m$.

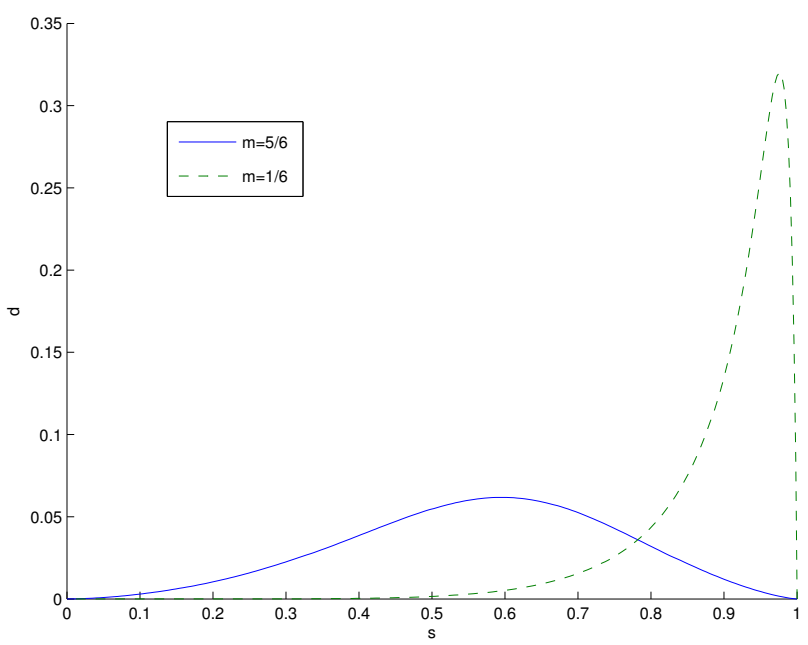

Figure 2. Diffusion coefficient, $d$, versus saturation, $s$, for two-phase flow. For all $m, d(0)=d(1)=0$. For small $m, \frac{d d}{d s}(1)=\infty$. Consequently, $d$ is not Lipschitz continuous at $s=1$ for small $m$.

there is no net movement of the fluid mixture and buoyancy and capillary pressure are the only driving forces. The case with $g_{x}=0$ is called capillary redistribution. For these physical constants with $g_{x}=-1$, the nonlinear coefficients for two-phase flow are plotted in figures 1 and 2 for large and small $\mathrm{m}$. The advective flux in figure 1 is zero at $s_{e}=0$ and $s_{e}=1$, but it has unbounded slope as $s_{e}$ approaches one. Likewise, the diffusion coefficient in figure 2 is zero at

Table 1. Summary of model parameters for $0<m<1$

\begin{tabular}{lccc} 
& $p$ & $q$ & $b_{0}$ \\
\hline Two-phase & $3 / 2+\mathrm{m}$ & $1 / 2+2 \mathrm{~m}$ & -1 \\
Richards & $1-\mathrm{m}$ & $\mathrm{m}$ & 1 \\
\hline
\end{tabular}

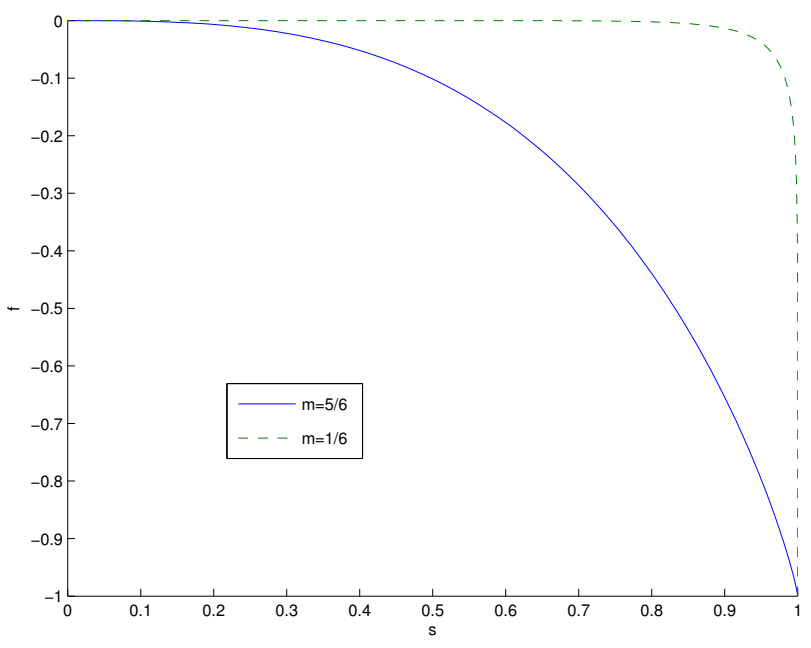

Figure 3. advective flux, $f$, versus saturation, $s$, for Richards' equation. For all $m, f(1)=-1$, and $\frac{d f}{d s}(1)=$ $-\infty$. Consequently, $f$ is not Lipschitz continuous at $s=1$.

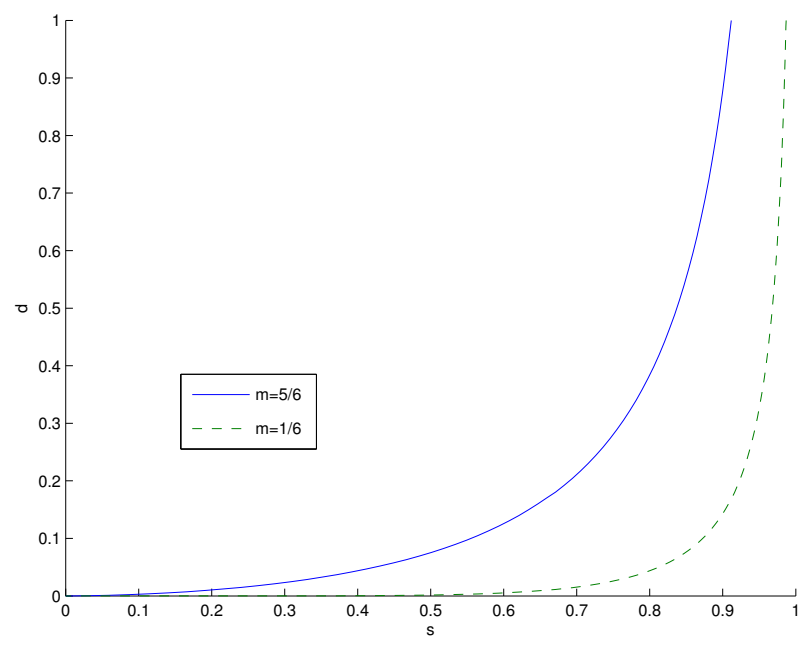

Figure 4. Diffusion coefficient, $d$ vs. $s$ for Richards' Equation. For all $m d(1)=\infty$ and $\frac{d d}{d s}(1)=\infty$. Thus, $d$ is neither bounded nor Lipschitz continuous with respect to $s$.

$s_{e}=0$ and $s_{e}=1$, but, for small $m$, the slope is unbounded as $s_{e}$ approaches one. The coefficients for Richards' equation are plotted in figures 3 and 4 . The diffusion coefficient itself is unbounded for Richards' equation. The advective flux is finite but has unbounded slope for all values of $m$ as $s_{e}$ approaches 1 .

\subsection{Asymptotic Approximations}

We would like to understand how the unbounded slope of $f$ and $d$, as well as the unboundedness of $d$ in Richards' equation, affect the solution behavior, and the speed of propagation, in particular. Since this behavior occurs only at $s_{e}=1$, we will use asymptotic approximations to $d$ and $f$ near $s_{e}=1$. For $u=1-s_{e}$ (i.e., non-wetting phase satura- 
tion) we have as $u \rightarrow 0$

$$
\begin{aligned}
\frac{d p_{c}}{d s} & =\frac{m-1}{m} \frac{1}{m}^{-m} u^{-m}+o\left(u^{-m}\right) \\
k_{r w} & =1-2 \frac{1}{m}^{m} u^{m}+o\left(u^{m}\right) \\
k_{r n} & =\frac{1}{m}^{2 m} u^{\frac{1}{2}+2 m}+o\left(u^{\frac{1}{2}+2 m}\right)
\end{aligned}
$$

Neglecting constants, we can approximate two-phase flow with the nonlinearities

$$
\begin{aligned}
d & =-\frac{k_{i}}{\omega \mu_{w}} \frac{m-1}{m} \frac{1}{m} u^{m} u^{\frac{1}{2}+m}+o\left(u^{\frac{1}{2}+m}\right) \approx u^{\frac{1}{2}+m} \\
f & ={\frac{k_{i}}{\omega \mu_{w}}}_{\frac{1}{m}}^{2 m} u^{\frac{1}{2}+2 m}\left(1-\frac{\rho_{n}}{\rho_{w}}\right) \rho_{w} g_{x}+o\left(u^{\frac{1}{2}+2 m}\right)
\end{aligned}
$$

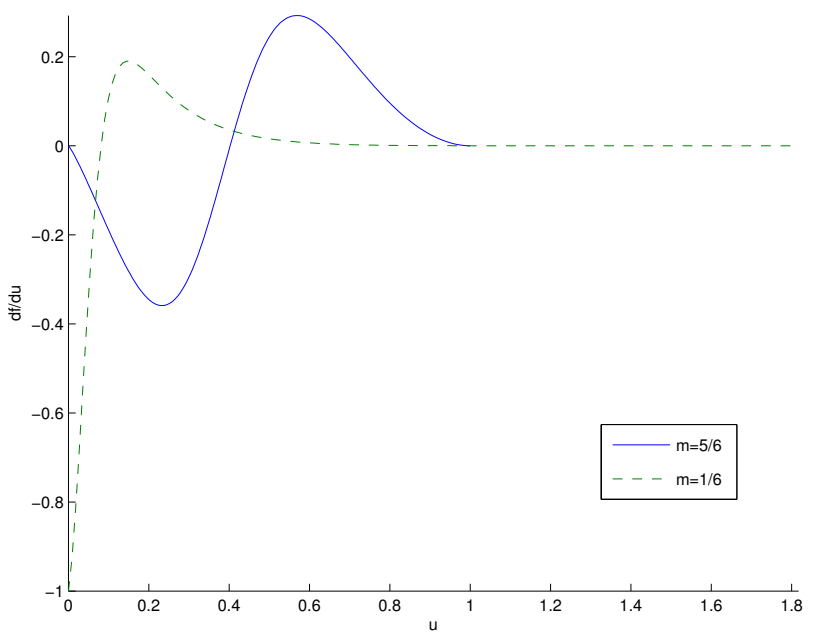

Figure 5. First derivative of advective flux, $d f / d v$, versus transformed variable $v$ for two-phase flow. Note that $d f / d v(0)=-1$ in agreement with the asymptotic approximation for $f(s)$.

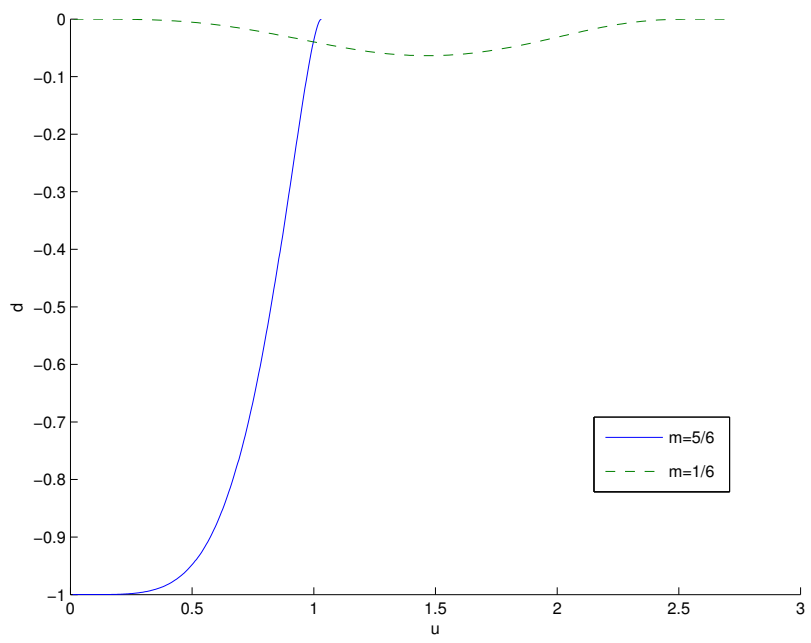

Figure 6. Diffusion coefficient, $d$, vs. transformed variable $v$ for Richards' equation. $d$ is now bounded and continuous but for $m<1 / 2 d(0)=0$. $\approx u^{\frac{1}{2}+2 m}$

and Richards' equation with

$$
\begin{aligned}
d & =\frac{k_{i}}{\omega \mu_{w}} \frac{m-1}{m} \frac{1}{m}{ }^{-m} u^{-m}+o\left(u^{-m}\right) \approx u^{-m} \\
f & =-\frac{k_{i}}{\omega \mu_{w}}\left(1-2 \frac{1}{m}^{m} u^{m}\right) \rho_{w} g_{x}+o\left(u^{m}\right) \\
& \approx 1-u^{m}
\end{aligned}
$$

Making these approximations yields the simple model

$$
u_{t}=\left(u^{p}\right)_{x x}+b_{0}\left(u^{q}\right)_{x}
$$

where $b_{0}=-1$ for two-phase flow and $b_{0}=1$ for Richards' equation. Note that we have used the Kirchoff transformation on the diffusion coefficient:

$$
u^{p}=\int_{0}^{u} d(u) d u
$$

In terms of the parameter $m$, the approximate two-phase flow model has $p=3 / 2+m$ and $q=1 / 2+2 m$ where the ranges of $p$ and $q$ are $1<p<5 / 2$ and $0<q<5 / 2$. Thus, $f$ has infinite slope at $s=1$ for $m<1 / 4$, which holds both for the simplified model and the original two-phase model. The approximate Richards' equation has $p=1-m$ and $q=m$ where the range of $p$ and $q$ is $0<p, q<1$. Thus $\left|\frac{d f}{d u}\right|$ blows up faster than $d$ when $m<1 / 2$, and we shall see that the speed of propagation changes from infinite to finite at this point. For the van Genuchten parameter $n$, this corresponds to the range $1<n<2$, which is a case studied previously in the context of numerical approximations Miller et al. [1998]. These relations are summarized in table 1 .

\subsection{Variable Transformation}

The asymptotic approximations above not only yield simplified model equations but also a change of variables for the original model equations that will yield once-differentiable coefficients over the entire range of saturations $0 \leq s_{e} \leq 1$.

First, note that if we use the Kirchoff transformation

$$
\phi(u)=\int_{0}^{u} d(u) d u
$$

then $\phi$ is once differentiable as long as $d$ is continuous. Hence only in the case of Richards' equation, where $d$ is unbounded, must we consider the behavior of $d$.

For two-phase flow in the case $m<1 / 4$, we will make the substitution

$$
v=\frac{1}{m}^{2 m} u^{\frac{1}{2}+2 m}
$$

This transformation yields nonlinear coefficients $s, f$, and $\phi$ that are differentiable in $v$. Figure 5 shows $d f / d v$. For $m \geq 1 / 4$ no variable transformation is necessary so we set $v=u$.

The variable transformations are slightly more complex for Richards' equation. For $m \geq 1 / 2, d \rightarrow \infty$ and $d /\left|\frac{d f}{d u}\right| \rightarrow$ $\infty$ as $u \rightarrow 0$. We design a change of variables $v(u)$ so that $\left(d p_{c} / d s\right)(d v / d u)(0)=-1$. Such a $v(u)$ will yield a diffusion coefficient that is bounded and continuous and, therefore, a potential $\phi$ that is differentiable and an advective flux that is zero at $u=0$. This consideration leads to

$$
v=m^{m} u^{1-m}
$$

For $m<1 / 2, d /\left|\frac{d f}{d u}\right| \rightarrow 0$ as $u \rightarrow 0$. Thus, as we did in the two-phase case, we construct a change of variables so that 
the advective flux is differentiable:

$$
v=2 \frac{1}{m}^{m} u^{m}
$$

For the simplified model equation we can use

$$
v=u^{\min (p, q)}
$$

These variable transformations do not change the characteristic speeds for the first-order part of the equation, which are

$$
\frac{d x}{d t}=\frac{\frac{\partial f}{\partial v}}{\frac{\partial s}{\partial v}}=\frac{\partial f}{\partial s}
$$

The shock speeds are also unchanged, since for a discontinuity with left state $s_{-}=s\left(v_{-}\right)$and right state $s_{+}=s\left(v_{+}\right)$ we have

$$
d=\frac{f\left(s_{+}\right)-f\left(s_{-}\right)}{s_{+}-s_{-}}=\frac{f\left(u_{+}\right)-f\left(u_{-}\right)}{s\left(u_{+}\right)-s\left(u_{-}\right)}
$$

We make these observations since, in the case of first-order conservation laws, not all transformations of the equations yield the same solution in the sense of entropy-satisfying weak solutions. In such cases two versions of an equation that are equivalent for classical solutions nevertheless have shock waves moving at different speeds for the same left and right states (c.f. [Smoller, 1991]). The simple variable transformations above yield equations with the same entropy-satisfying weak solutions as the original equation. On the other hand, the diffusion coefficient (in the sense of $d \phi / d v)$ can change dramatically in the transformed equation, and the way it changes yields additional insight into the character of solutions. For Richards' equation the diffusion coefficient of the transformed equation $(m<1 / 2)$ is now zero at $s=1(u=0)$, as is shown in figure 6 .

In the next section we apply the results of Gilding and Kersner [1996] to characterize the models above with respect to the speed of propagation.

\section{Speed of Propagation}

\subsection{Simple Model}

Conveniently, Gilding and Kersner [1996] provide a corollary to their main results which describes the speed of propagation of the simple nonlinear advection-diffusion-reaction equation

$$
u_{t}=\left(u^{p}\right)_{x x}+b_{0} u_{x}^{q}+c_{0} u^{r}
$$

over a wide range of values of $p, q, r, b_{0}$, and $c_{0}$. The relevant portion of this corollary states that for $c_{0}=0$ and $b_{0} \neq 0$, equation 32 has finite speed of propagation if and only if

1. $p>1$ and $q \geq 1$, or

2. $q<1, b_{0}>0$ and $p>q$

For the simplified two-phase flow model, the first case holds when $m \geq 1 / 4$ while the second case holds for $g_{x}>0$. Thus the solution will display infinite speed of propagation when $g_{x}=-1$ and $m<1 / 4$, in other words, in the countercurrent flow problem when the advective flux has infinite slope at $s_{e}=1$. For the simplified Richards' equation model, only the second case holds and then only when $m<1 / 2$.

\subsection{Two-Phase Flow and Richards' Equation}

Since we are considering the behavior near $s_{e}=1$ we consider the equation in terms of $u=1-s_{e}$

$$
u_{t}=\left[a(u)_{x}+b(u)\right]_{x}
$$

where

$$
a(u)=\int_{0}^{u} d(1-w) d w
$$

The formula for $d$ is given by equation 5 or equation 8 . For two-phase flow we have $b=f(1-u)$ where $f$ is given by equation 6 . For Richards' equation we take $b=f(1-u)+1$ where $f$ is given by equation 7 . With these definitions equation 33 is equivalent to equation 4 and $a(0)=b(0)=0$, which is the form of the equation needed to apply the following two results from Gilding and Kersner [1996].

Theorem 1 (Gilding and Kersner) The equation

$$
u_{t}=[a(u)]_{x x}
$$

displays finite speed of propagation if and only if

$$
\int_{0}^{\delta} \frac{1}{w} d a(w)<\infty \text { for some } \delta \in(0, \infty)
$$

where the integral is in the sense of Lebesgue-Stieltjes. Since $a$ is differentiable on $(0, \delta), d a(w)=d(1-w) d w$. For two-phase flow we see from equation 19 that equation 36 reduces to

$$
\int_{0}^{\delta} \frac{C w^{\frac{1}{2}+m}+o\left(w^{\frac{1}{2}+m}\right)}{w} d w<\infty \quad \forall \delta>0
$$

so that in the case of capillary redistribution two-phase flow has finite speed of propagation for all $m$. For Richards' equation we see from equation 21 that equation 36 reduces to

$$
\int_{0}^{\delta} \frac{C w^{-m}+o\left(w^{-m}\right)}{w} d w=\infty \quad \forall \delta>0
$$

so that in the case of capillary redistribution, Richards' equation has infinite speed of propagation for all $m$.

When $g_{x} \neq 0$ we have

Theorem 2 (Gilding and Kersner) The equation

$$
u_{t}=\left[a(u)_{x}+b(u)\right]_{x}=0
$$

displays finite speed of propagation if and only if

$$
\max \{-b(w), 0\}=O(w) \text { as } w \downarrow 0
$$

and

$$
\int_{0}^{\delta} \frac{1}{\max \{b(w), w\}} d a(w)<\infty \text { for some } \delta \in(0, \infty)
$$

For two-phase flow we see from equation 20 that equation 40 fails when $m<1 / 4$. When $m \geq 1 / 4$ then equation 41 reduces to equation 37 so that two-phase flow displays finite speed of propagation in the gravity segregation case if and only if $m \geq 1 / 4$.

For Richards' equation, equation 40 holds for $0<m<1$ while equation 41 reduces to

$$
\int_{0}^{\delta} K w^{-2 m}+o\left(w^{-2 m}\right) d w
$$

Table 2. Summary of Finite Speed of Propagation Results

\begin{tabular}{lcc} 
& Capillary Redistribution & Gravity Segregation \\
\hline Two-phase & $0<m<1$ & $1 / 4 \leq m<1$ \\
Richards & - & $0<m<1 / 2$ \\
\hline
\end{tabular}


Hence Richards' equation displays finite speed of propagation in the gravity segregation case if and only if $m<1 / 2$.

These results are in agreement with those for the simple model and are summarized in table 2 .

\section{Analytical Solutions}

In the following sections we examine solutions with and without finite speed of propagation by looking at special solutions.

\subsection{First-Order Approximation}

First we consider solutions of the first-order approximation of equation 4

$$
s_{t}+f_{x}=0
$$

on the spatial domain $(-\infty, \infty)$ given Riemann initial data,

$s(x, 0)=\left\{\begin{array}{lll}s_{-} & \text {for } & x<0 \\ s_{+} & \text {for } & x \geq 0\end{array}\right.$

Equation 43 is usually referred to as the Buckley-Leverett equation when $f$ is given by equation 6 . This equation generally only has weak solutions, which may be discontinuous and which furthermore are not unique (c.f. [Evans, 1998; Leveque, 1990]). When the solution fails to be continuous, a discontinuity with left state $s_{-}$and right state $s_{+}$must propagate with speed

$$
c=\frac{f\left(s_{+}\right)-f\left(s_{-}\right)}{s_{+}-s_{-}}
$$

Unique weak solutions can be specified by requiring that discontinuous solutions be in some sense a limit of solutions of equation 43 with a second-order regularization term $\epsilon u_{x x}$ (a vanishing viscosity solution). For non-convex $f$, this requirement can be stated as the Oleinik entropy condition [Oleinik, 1957] (c.f. [Osher, 1984; Leveque, 1990])

$$
\frac{f(s)-f\left(s_{-}\right)}{s-s_{-}} \geq c \geq \frac{f(s)-f\left(s_{+}\right)}{s-s_{+}}
$$

for all $s$ between $s_{-}$and $s_{+}$. For a general Riemann problem with $s_{-}<s_{+}$, the unique solution can be determined from the convex hull of the set

$$
\left\{(s, y) \mid s_{-} \leq s \leq s_{+} \text {and } y \geq f(s)\right\}
$$

The convex hull will consist of the graph of $f$ and some set of chords enclosing regions where $f$ is concave down. A chord on the boundary of the convex hull represents two values of the solution connected by a shock wave, and the slope of the chord is the shock speed $c$. We are interested in the counter-current flow problem where $s_{+}=1$. For two-phase flow we see from figure 1 that the solution will have at least one shock wave connecting to $s_{+}=1$ and moving to the right $(c>0)$ when $m$ is large, specifically when $m>1 / 4$. On the other hand, when $m$ is small, the solution near $s_{+}$ will be a rarefaction wave with the value $s_{+}=1$ traveling at infinite speed to the right. Thus, in this case, the loss of finite speed of propagation occurs even in the first-order approximation.

Solutions for $s_{+}=1$ and $s_{-}=0$ are given in figure ?? corresponding to each case. As can be seen from figure 3, Richards equation always has solutions consisting of a single shock traveling to the left because the flux is convex down for all $m$. The solution for $s_{+}=1$ and $s_{-}=0$ is simply a shock wave with $c=-1$. Although we only consider this case at length, we can briefly mention a few other cases.

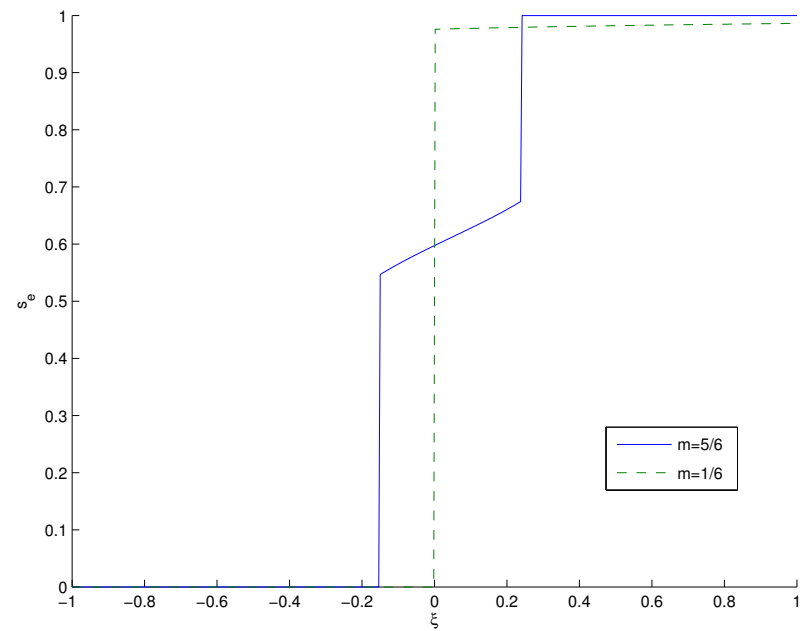

Figure 7. Riemann solution for the Buckley-Leverett equation for the cases $m=5 / 6$ and $m=1 / 6$.

For example, if we consider values of $s_{-}$close to $s_{+}$we can produce a shock wave moving arbitrarily fast. Furthermore, if we consider the initial data reversed (also known as capillary rise) we again obtain a rarefaction wave with values of $s_{-}=1$ moving left at infinite speed: the saturated region desaturates instantaneously.

For completeness we note that the solution in figure ?? was computed automatically by solving the optimization problem [Osher, 1984]

$$
s(\xi)=\operatorname{argmin}_{s \in\left[s_{-}, s_{+}\right]}[f(s)-\xi s]
$$

where $\xi=x / t$. This formula is a concise statement of the entropy satisfying weak solution to the Riemann problem when $s_{+} \geq s_{-}$(a similar formula holds for $\left.s_{+} \leq s_{-}\right)$. This problem was solved in MATLAB using the fminbnd routine with tolerances of $1.0 e-5$.

\subsection{Traveling Wave Solutions}

There are two reasons why studying the first-order problem above is not sufficient for understanding the solution dynamics of the full second-order model. The first is that a second-order term can destroy the finite speed of propagation property. The second is that, even without a first-order term, the equation can exhibit finite speed of propagation. In this section we will see how the full second-order model has finite speed of propagation (in some cases) by examining traveling wave solutions.

The solutions in the previous section are the unique entropy satisfying weak solutions composed of continuous rarefaction waves and discontinuous shock waves. The former condition arises due to the conservation form of the equation while the latter can be derived, as mentioned above, by requiring that any moving discontinuity correspond to traveling wave solutions of a second-order regularization of the equation. In short, we should already expect that the only traveling waves that arise in the full model are the shock waves that arise in the simplified model. In order for the solutions to exhibit finite speed of propagation, the front $\zeta(t)$ must be finite for $0<t<\tau$.

To derive traveling wave solutions, we assume that the solution has the form $v(x, t)=v(x-c t)=v(\xi)$ where $c$ is the wave speed (to be determined). Using the variable transformations above, we can consider $s, f$, and $\phi$ to be $C^{1}$ 
functions of $v$. Making this substitution into Equation 4 yields

$$
-c s_{\xi}+f_{\xi}-\phi_{\xi \xi}=0
$$

Integrating once with respect to $\xi$ and rearranging yields

$$
\phi_{\xi}=-c s(\xi)+f(\xi)+b
$$

where $b$ is the constant of integration. We will enforce the following asymptotic boundary conditions on equation 50

$$
\begin{aligned}
\lim _{\xi \rightarrow \pm \infty} v & =v_{ \pm} \\
\lim _{\xi \rightarrow \pm \infty} \phi_{\xi} & =0
\end{aligned}
$$

and write $f_{ \pm}$for $f\left(v_{ \pm}\right)$and $s_{ \pm}$for $s\left(v_{ \pm}\right)$. These boundary conditions require

$$
\lim _{v \rightarrow v_{ \pm}}-c s+f+b=0
$$

which implies

$$
b=c s_{-}-f_{-}=c s_{+}-f_{+}
$$

From equation 54 we can also determine $c$

$$
c=\frac{f_{+}-f_{-}}{s_{+}-s_{-}}
$$

Note that equation 55 is the Rankine-Hugoniot condition. We will take $b=c s_{+}-f_{+}$. The traveling wave equation is then

$$
\begin{aligned}
\phi(v)_{\xi} & =f(v)-\left\{\frac{f_{+}-f_{-}}{s_{+}-s_{-}}\left[s(v)-s_{+}\right]+f_{+}\right\} \\
& =h\left(v_{-}, v_{+}, v\right)
\end{aligned}
$$

Equation 56, with boundary conditions given by equations 51 and 52, may not have a solution, and, if it does, solutions are not unique (if $v(\xi)$ is a solution, then $v\left(C\left(\xi-\xi_{0}\right)\right.$ ) is also a solution). For two-phase flow and Richards' equation and the variable transforms we have defined, $\phi(v)$ is a monotone decreasing function of $v$. Since we consider the case $v_{+}=0$ and $0<v_{-}<1$, this implies that $\phi\left(v_{-}\right)<\phi\left(v_{+}\right)$. The asymptotic boundary conditions can only be met if $h>0$ for $v_{+} \leq v \leq v_{-}$. The term in braces on the right-hand side of equation 56 is an expression for the chord connecting $f_{+}$and $f_{-}$. The requirement that $h>0$ can then be interpreted as a constraint that the chord connecting $f_{+}$and $f_{-}$ lie below the graph of $f(s)$. Rearranging the requirement that $h>0$ yields half the Oleinik entropy inequality above. The other half comes from using the second definition of the constant $b$ above. Thus, the analysis of the first-order case carries over to the full model in the following way: traveling wave solutions exist whenever the solution of the Riemann problem consists of a single shock wave. Traveling wave solutions exist for the two-phase model when $m>1 / 4$ and in all cases for the Richards' equation model.

The question of whether the support of $v(x, t)$ has an upper bound for $t>0$ (i.e., that the solution has finite speed of propagation) can then be answered by determining when the solution of equation 56 with initial condition $v_{-}>0$ goes to $v_{+}$for $\xi<\infty$. Dividing equation 56 by its (positive) right hand side and integrating once yields

$$
G(v)=\int_{v_{0}}^{v} \frac{\frac{d \phi}{d v}}{f(v)-\left[c\left(s(v)-s_{+}\right)-f_{+}\right]} d v=\xi
$$

where $v_{0}=v(0)$ can be chosen arbitrarily in $\left(v_{+}, v_{-}\right)$. Thus, if the integral in equation 58 is bounded as $v \rightarrow v_{+}, v(\xi)$ goes to $v_{+}$in "finite $\xi$ " and the front $\zeta(t)$ is given by $t G\left(v_{+}\right)$.

Consider first the simplified model problem with $u_{-}=1$, $u_{+}=0$. The integral above is

$$
\xi=\int_{u_{0}}^{u} \frac{p u^{p-1}}{-b_{0} u^{q}-c u} d u
$$

If $b_{0}=-1, p=1$ and $q=2$, the simplified model is viscous Burgers' equation, and we have

$$
\xi(u)=\log (1-u)-\log (u)
$$

Since $\xi(u)$ is unbounded as $u \rightarrow u_{ \pm}$, we see that the solution has infinite speed of propagation. On the other hand, for $p=2$, we have

$$
\xi(u)=2 \log (1-u)
$$

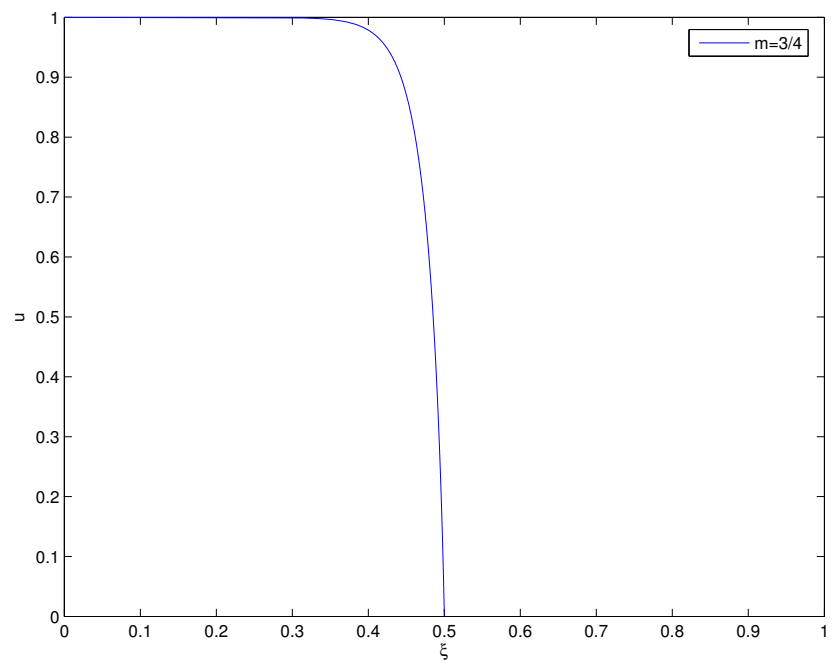

Figure 8. Traveling wave solution of the simplified twophase flow model.

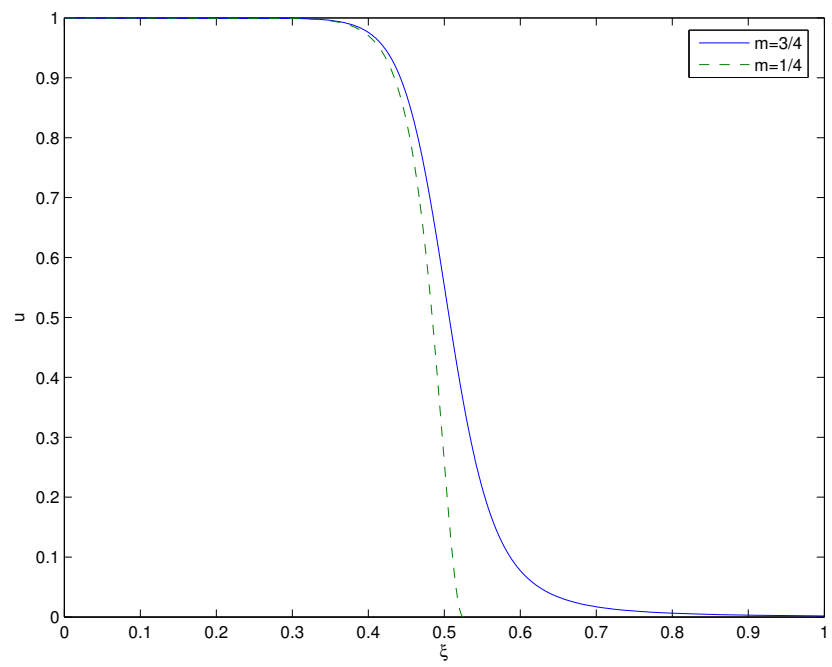

Figure 9. Traveling wave solutions of the simplified Richards' equation model. Note that the wave speed is negative, and for $m>1 / 2$ the support is unbounded. 
and therefore $\zeta(t)=2 t$. Note also that $\frac{d \xi}{d u}=-2 /(1-u)$ and therefore $-1 / 2 \leq \frac{d u}{d \xi} \leq 0$ : solutions are Lipschitz continuous. For $p=3$ we have

$$
\xi(u)=3(u+\log (1-u))
$$

so that $\zeta(t)=3 t$ and the speed of propagation is again finite. However $\frac{d \xi}{d u}=3[1-1 /(1-u)]$ and therefore $\frac{d u}{d \xi} \rightarrow-\infty$ as $x \rightarrow \zeta(t)$ : solutions fail to be Lipschitz continuous in space or time at $\zeta(t)$.

For two-phase flow we know $q>1$ if $m>1 / 4$, so we can write the inverse of the solution as

$$
\xi=\int_{u_{0}}^{u} \frac{p}{u^{2-p}\left(u^{q-1}-1\right)} d u
$$

Since $p>1$ this implies that the front for the traveling wave solutions to two-phase flow move with finite speed for all cases with $m>1 / 4$, in agreement with the theory.

For the full two-phase model there is no traveling wave solution for the case when $m \leq 1 / 4$. For $m>1 / 4$ the traveling wave solution is bounded, and for $m>1 / 2$ the derivative of the solution blows up at the front. We can consider equations 61 and 62 as approximate models for the behavior of two-phase flow near $s=1$. The traveling wave solution for $m=3 / 4$ is given in figure 8 ( $\xi$ is shifted and scaled).

For Richards' equation, on the other hand, we have $0<p, q<1, p=1-q(q=m)$, and we can see by rearranging the integrand of equation 59 as

$$
\begin{aligned}
\xi & =\int_{u_{0}}^{u} \frac{p}{u^{2(1-p)}\left(1-u^{1-q}\right)} d u \\
& =\int_{u_{0}}^{u} \frac{1-m}{u^{2 m}\left(1-u^{1-m}\right)} d u
\end{aligned}
$$

that we have infinite speed of propagation when $m \geq 1 / 2$. When $m=1 / 2$ we have

$$
\xi(u)=\log (1-\sqrt{u})-\log (\sqrt{(u)})
$$

When $m=1 / 4$ we have

$$
\xi(u)=\frac{1}{2 u^{1 / 2}}+\frac{1}{u^{1 / 4}}-\log \left(u^{1 / 4}\right)+\log \left(1-u^{1 / 4}\right)
$$

When $m=3 / 4$ we have

$$
\begin{aligned}
\xi(u)= & \log \left(1-u^{1 / 4}\right)-\log \left(u^{1 / 2}+u^{1 / 4}+1\right) / 2 \\
& +3^{1 / 2} \arctan \left(1 / 3\left(2 u^{1 / 4}+1\right) 3^{1 / 2}\right)
\end{aligned}
$$

Traveling wave solutions for $m=1 / 4$ and $m=3 / 4$ are given in figure 9 ( $\xi$ is shifted and scaled).

\subsection{Barenblatt's Solution}

The traveling waves in the previous section displayed both finite and infinite speed of propagation. As was seen in the calculations, the boundedness of the traveling wave depends mainly on the ratio of diffusion and advection. When advection is absent, the equation can still have finite speed of propagation and therefore moving fronts. We now consider the simplified model given in equation 23 with $g=0$ and $q_{t}=0$ (i.e. no advection):

$$
u_{t}-\left(u^{p}\right)_{x x}=0
$$

By seeking a solution of the form $u(x, t)=t^{-\alpha} v\left(x t^{-\beta}\right)$ it can be shown that a solution on $(-\infty, \infty)$ is given by (c.f.
[Evans, 1998])

$$
u(x, t)=\frac{1}{t^{\frac{1}{p+1}}}\left[b-\frac{p-1}{2 p(p+1)}\left(\frac{x}{t^{\frac{1}{p+1}}}\right)^{2}\right]^{\frac{1}{p-1}} \quad|x|<\zeta(t)
$$

for $p \neq 1$, where $b$ is determined from the particular initial conditions $u(x, 0)=M \delta(x)$, and where $\delta$ is the Dirac delta function. For $p>1$ the front location is given by

$$
\zeta(t)=\left(\frac{2 p(p+1)}{p-1} b\right)^{1 / 2} t^{\frac{1}{p+1}}
$$

Thus the propagation speed is finite for $p>1$, for all $t$. Note also that $\left|u_{t}\right|$ and $\left|u_{x}\right|$ blow up at $\zeta(t)$ if $p>2$. The case $p>2$ corresponds to two-phase flow with $m>1 / 2$. The case where $p<1$ corresponds to Richards' equation. Since in this case $p-1<0$, we have that $\zeta=\infty$ and the speed of propagation is infinite (the solution is positive for all $x$ for $t>0)$. Graphs of the solution are given in figure 10 .

\section{Numerical Solutions}

We are capable of writing closed-form solutions in only a few of the instances above. We were able to demonstrate the effect of infinite speed of propagation in a few special cases of approximate model equations. To look at the more general cases we need to use numerical solutions.

We will look at only simple discretization methods here: forward and backward Euler in time for fixed time step and central and upwind finite differences in space. For the domain $[0,1] \times[0, T]$ the discrete solution is defined over the spatial grid $x_{i}=i h, i=0, \ldots, n, h=1 / n$ at discrete times $t^{j}=j k, j=0, \ldots, m, k=T / m$. We consider first Dirichlet boundary conditions $s(0)=s(1)=S$ so that a general fully discrete form of the equation is, $i=1, \ldots, n-1$, $\mathbf{s}=\left(s_{0}, \ldots, s_{n}\right)$

$$
\begin{aligned}
s_{i}^{j}= & s_{i}^{j-1}+\frac{k}{h}\left\{\left[\frac{\phi\left(s_{i+1}^{*}\right)-\phi\left(s_{i}^{*}\right)}{h}-F_{i+1 / 2}\left(\mathbf{s}^{*}\right)\right]\right. \\
& \left.-\left[\frac{\phi\left(s_{i}^{*}\right)-\phi\left(s_{i-1}^{*}\right)}{h}-F_{i-1 / 2}\left(\mathbf{s}^{*}\right)\right]\right\}
\end{aligned}
$$

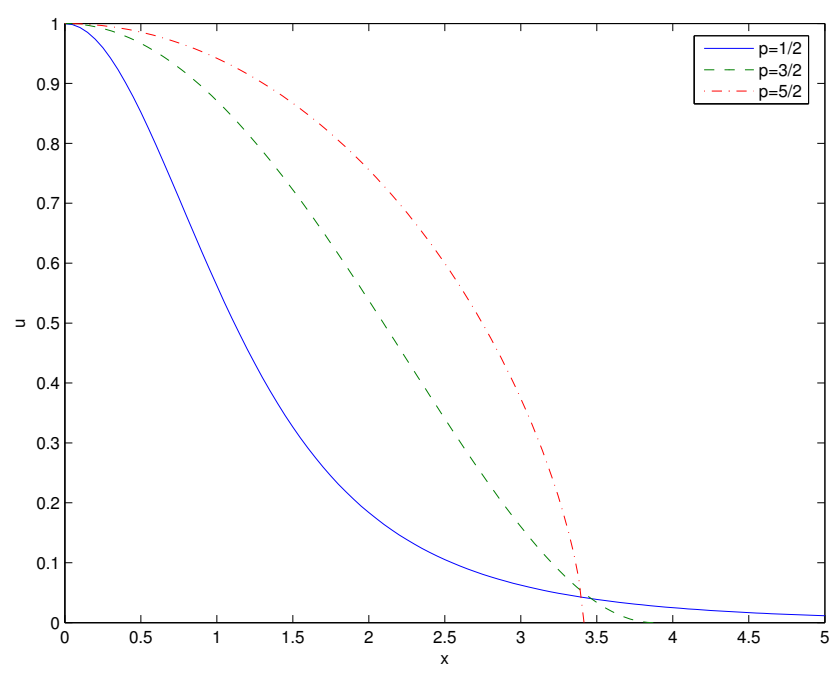

Figure 10. Barenblatt's Solution 
First we choose $*=n$ and the numerical flux $F$ as

$$
F_{i+1 / 2}(\mathbf{s})=\frac{f\left(s_{i}\right)+f\left(s_{i+1}\right)}{2}
$$

Consider initial conditions $s(x, t)=S+w(x, t)$ where $w(x, t)$ is a small perturbation. Linearizations about $S$ are given by

$\phi(s) \approx \phi(S)+\frac{d \phi}{d s}(S)(s-S)=\phi(S)+D(s-S)$

$f(s) \approx f(S)+F(s-S)$

where $\frac{d \phi}{d s}(S)=D$ and $\frac{d f}{d s}(S)=F$. Substituting these linearizations for the nonlinear coefficients yields the standard fully discrete linear advection-diffusion equation for $i=1, \ldots, n-1$

$$
s_{i}^{j}=\left(\frac{D k}{h^{2}}-\frac{F k}{2 h}\right) s_{i+1}^{j-1}+\left(1-\frac{2 D k}{h^{2}}\right) s_{i}^{j-1}+\left(\frac{D k}{h^{2}}+\frac{F k}{2 h}\right) s_{i-1}^{j-1}
$$

For periodic boundary conditions the standard Von Neumann stability analysis shows that the method is stable for time steps $k>0$ satisfying (c.f. [Strikwerda, 1989; Hundsdorfer and Verwer, 2003])

$$
k \leq \frac{h^{2}}{2 D}=k_{0}
$$

If $h$ is then chosen such that

$$
h \leq \frac{2 d}{|F|}=h_{0}
$$

then

$$
\begin{aligned}
\max _{i}\left|s_{i}^{j}\right| \leq & \max _{i}\left\{\left|\left(\frac{D k}{h^{2}}-\frac{F k}{2 h}\right) s_{i+1}^{j-1}\right|\right. \\
& +\left|\left(1-\frac{2 D k}{h^{2}}\right) s_{i}^{j-1}\right| \\
& \left.+\left|\left(\frac{D k}{h^{2}}+\frac{F k}{2 h}\right) s_{i-1}^{j-1}\right|\right\} \\
\leq & \max _{i}\left\{\left(\frac{D k}{h^{2}}-\frac{F k}{2 h}\right)\left|s_{i+1}^{j-1}\right|\right. \\
& +\left(1-\frac{2 D k}{h^{2}}\right)\left|s_{i}^{j-1}\right| \\
& \left.+\left(\frac{D k}{h^{2}}+\frac{F k}{2 h}\right)\left|s_{i-1}^{j-1}\right|\right\} \\
\leq & \max _{i}\left|s_{i}^{j-1}\right|
\end{aligned}
$$

and, therefore, saturations will remain in the physical range $0 \leq s \leq 1$.

From equations 19-22 we have for two-phase flow

$k_{0} \approx \frac{h^{2}}{2 u^{\frac{1}{2}+m}}$

$h_{0} \approx \frac{u^{\frac{1}{2}+m}}{\left(\frac{1}{2}+2 m\right) u^{2 m-\frac{1}{2}}}$

and for Richards' equation

$k_{0} \approx \frac{h^{2}}{2 u^{-m}}$

$h_{0} \approx \frac{u^{-m}}{(1-m) u^{m-1}}$

which yields the following three cases:

1. (Two-phase Flow) $h_{0} \rightarrow 0$ as $S \rightarrow 1$.
2. (Richards' equation for $m \geq 1 / 2) k_{0} \rightarrow 0$ as $S \rightarrow 1$ but $h_{0}$ is bounded away from zero.

3. (Richards' equation with $m<1 / 2$ ) $h_{0} \rightarrow 0$ as $S \rightarrow 1$.

Since $k_{0} \rightarrow 0$ as $h_{0} \rightarrow 0$ the discretization is essentially unusable for these model equations. If we use an upwind advection discretization given by

$$
F_{i+1 / 2}(\mathbf{s})= \begin{cases}F s_{i} & \text { if } F>0 \\ F s_{i+1} & \text { if } F<0\end{cases}
$$

then the discrete maximum principle holds [Strikwerda, 1989] if

$$
\frac{2 D k}{h^{2}}+\frac{F k}{h} \leq 1
$$

This condition can be met only in the two-phase flow case with $m \geq 1 / 4$. We therefore elect to use backward Euler with upwinding:

$$
s_{i}^{j}=s_{i}^{j-1}+\frac{D k}{h^{2}} s_{i+1}^{j}-\left(\frac{2 D k}{h^{2}}+\frac{F k}{h}\right) s_{i}^{j}+\left(\frac{D k}{h^{2}}+\frac{F k}{h}\right) s_{i-1}^{j}
$$
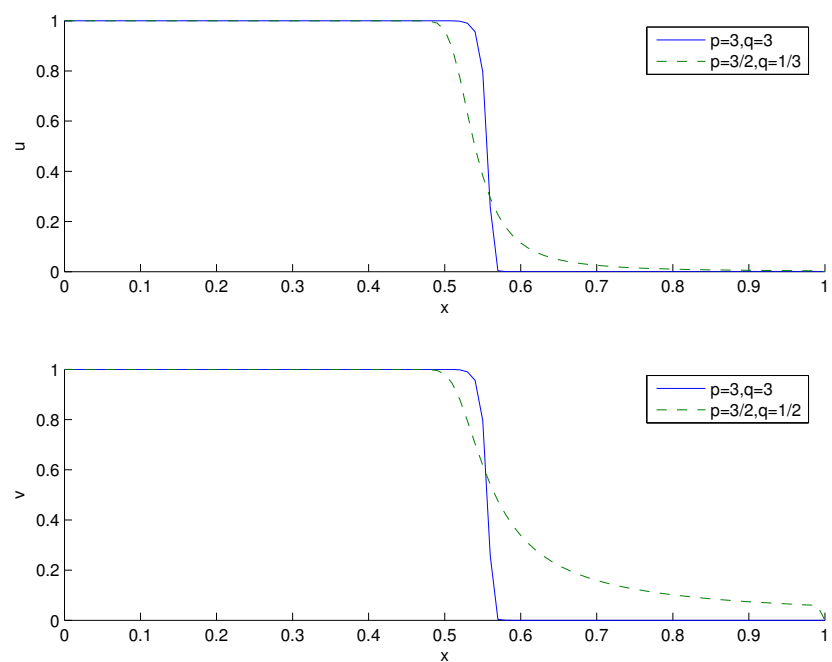

Figure 11. Solutions of the simplified two-phase model at $t=0.05$.
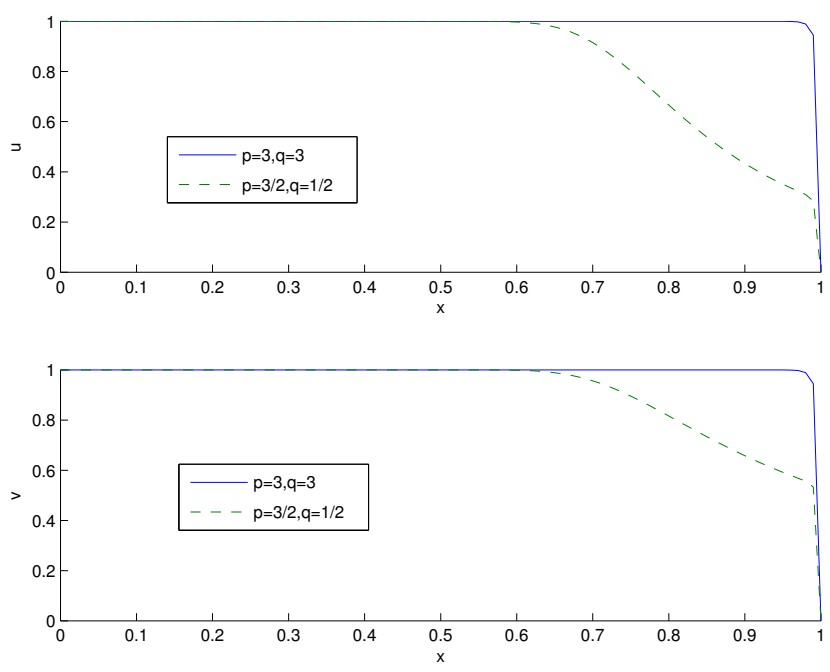

Figure 12. Solutions of the simplified two-phase model at $t=0.5$ 
Taking absolute values and assuming $F>0$ yields

$$
\begin{aligned}
\left(1+\frac{2 D k}{h^{2}}+\frac{F k}{h}\right)\left|s_{i}^{j}\right|= & \left|s_{i}^{j-1}+\frac{D k}{h^{2}} s_{i+1}^{j}+\left(\frac{D k}{h^{2}}+\frac{F k}{h}\right) s_{i-1}^{j}\right| \\
\leq & \left|s_{i}^{j-1}\right|+\frac{D k}{h^{2}}\left|s_{i+1}^{j}\right| \\
& +\left(\frac{D k}{h^{2}}+\frac{F k}{h}\right)\left|s_{i-1}^{j}\right|
\end{aligned}
$$

Thus we have unconditionally

$$
\max _{i}\left|s_{i}^{j}\right| \leq \max _{i}\left|s_{i}^{j-1}\right|
$$

Since backward Euler is fully implicit, we must solve a system of nonlinear algebraic equations at each time step of the form $G\left(s^{j}\right)=0$. Standard theories for the convergence of Newton's method do not apply when $G$ is not Lipschitz continuous, which is the case when $m<1 / 4$ for two-phase flow or $m<1 / 2$ for Richards' equation [Kelley, 1995; Qi and Sun, 1993]. We therefore apply the change of variables as described above to solve the equivalent root-finding problem $G\left(v^{j}\right)$. This equivalent problem is Lipschitz continuous in $v$, and, therefore, the convergence theory for non-smooth Newton methods is applicable.

In computations with the two-phase and Richards' models, $\phi(v)$ is approximated with a piecewise linear spline. To compute the spline approximation we set $v_{l}=l \Delta v$ where $\Delta v=\max v /(n s-1)$ and $n s$ is the number of spline knots. We then set $\phi_{0}=0$ and approximate $\phi$ recursively as

$$
\phi_{l}=\phi_{l-1}+\Delta v d\left(v_{l}\right) \quad l=1, \ldots, n s
$$

In the nonlinear context we use the following simple generalization of the upwind formula

$$
F_{i+1 / 2}(\mathbf{s})= \begin{cases}f\left(s_{i}\right) & \text { if } \frac{f_{i+1}-f_{i}}{s_{i+1}-s_{i}}>0 \\ f\left(s_{i+1}\right) & \text { if } \frac{f_{i+1}-f_{i}}{s_{i+1}-s_{i}}<0\end{cases}
$$

This flux is not generally appropriate for the first-order approximation to two-phase flow (Buckley-Leverett), since the flux is non-convex. It is not entropy satisfying in the case of transonic rarefaction waves [Leveque, 1990].

The resulting nonlinear system was solved with Newton's method to a relative residual tolerance of $10^{-5}$. Figures $11-$ 14 are plots of the solution to the simple model for parameters corresponding to two-phase flow and Richards' equation. Figures $15-16$ are plots of the solution to the two-phase flow model at $t=0.05$ and $t=0.5 m=1 / 5$ and $m=5 / 6$. The consequence of infinite speed of propagation is the thin wedge of saturation that extends all the way to the right end of the domain as opposed to the sharp moving boundary that forms in the finite-speed case. Similar behavior is seen in the solutions of Richards' equation shown in figures 17-18. The sharp boundary traveling to the left in both two-phase flow and Richards' equation is a consequence of finite speed of propagation of wetting phase into non-wetting phase (the degeneracy at $s_{e}=0$ ).

\section{Conclusions}

We have considered two models of two-phase flow and the the closure relations presented in Parker et al. [1987], which are based on the relative permeability model of Mualem [1976] and the capillary pressure model of van Genuchten [1980]. For horizontal capillary redistribution (zero gravity and zero total fluid velocity), the full two-phase model displays finite speed of propagation. Richards' equation displays infinite speed of propagation in this case.

For two-phase flow in the case of counter-current flow with the pore size distribution index $m<1 / 4(n<4 / 3)$, non-wetting phase propagates into wetting phase-saturated regions with infinite speed. In this case, infinite speed of propagation is a consequence of the unbounded derivative of the advective flux. At $s_{w}=1$, the diffusion coefficient is degenerate while the characteristic speeds of the underlying first-order equation are infinite. A standard upwind advection discretization with forward Euler time stepping fails to have a discrete maximum principle in this case, while backward Euler with upwinding has no such requirement.

For Richards' equation, in the case of counter-current flow with $m<1 / 2(n<4 / 3)$, air propagates with finite speed. As in the degenerate case of two-phase flow, however, the standard upwind advection discretization with forward Euler time stepping fails to have a discrete maximum principle. Again, the backward Euler method has no such requirement.

Non-Lipschitz continuous closure relations for two-phase flow and Richards' equation both cause non-trivial differences in the qualitative behavior of solutions and in the difficulty of obtaining numerical solutions. While variable transformations can be used to derive equivalent models with smooth coefficients, the most important numerical difficulties arise from the properties of the solution itself and are therefore unaffected by the reformulation.

Acknowledgments. This work was supported by the Army Research Office, grant DAAD19-02-1-0391.

\section{References}

Bear, J. (1972), Dynamics of Fluids in Porous Media, Elsevier.

Chavent, G., and J. Jaffré (1986), Mathematical Models and Finite Elements for Reservoir Simulation., Studies in Mathematics and its Applications, vol. 17, Elsevier.

Evans, L. C. (1998), Partial Differential Equations, Graduate Studies in Mathematics, vol. 19, American Mathematical Society.

Gilding, B. H., and R. Kersner (1996), The characterization of reaction-convection-diffusion processes by traveling waves, Journal of Differential Equations, 124, 27-79.

Hundsdorfer, W., and J. Verwer (2003), Numerical Solution of Time-Dependent Advection-Diffusion-Reaction Equations, Springer.

Kelley, C. T. (1995), Iterative Methods for Linear and Nonlinear Equations, Frontiers in Applied Mathematics, vol. 16, SIAM.

Leveque, R. J. (1990), Numerical Methods for Conservation Laws, Birkhauser.

Miller, C. T., G. A. Williams, C. T. Kelley, and M. D. Tocci (1998), Robust solution of richards' equation for nonuniform porous media, Water Resources Research, 34(10), 2599-2610.

Mualem, Y. (1976), A new model for predicting the hydraulic conductivity of unsaturated porous media, Water Resources Research, 12(3), 513-522.

Oleinik, O. (1957), Discontinuous solutions of nonlinear differential equations, American Mathematical Society Translations Series 2., 26, 95-172.

Osher, S. (1984), Riemann solvers, the entropy condition, and difference approximations, SIAM Journal on Numerical Analysis, 21(2), 217-235.

Parker, J. C., R. J. Lenhard, and T. Kuppusamy (1987), A parametric model for constitutive properties governing multiphase flow in porous media, Water Resources Research, 23(4), 61824 .

Qi, L., and J. Sun (1993), A nonsmooth version of Newton's method, Mathematical Programming, 68, 353-367.

Richards, L. (1931), Capillary conduction of liquids through porous media, Physics, 1, 318-333.

Smoller, J. (1991), Shock Waves and Reaction-Diffusion Equations, Springer-Verlag.

Strikwerda, J. C. (1989), Finite Difference Schemes and Partial Differential Equations, Wadsworth \& Brooks/Cole. 
van Genuchten, M. T. (1980), A closed-form equation for predicting the hydraulic conductivity of unsaturated soils, Soil Science Society of America Journal, 44, 892-898.

C. E. Kees, Center for Research in Scientific Computation, Department of Mathematics, Box 8205 North Carolina State University, Raleigh, NC 27695-8205, USA. (chris_kees@ncsu.edu)
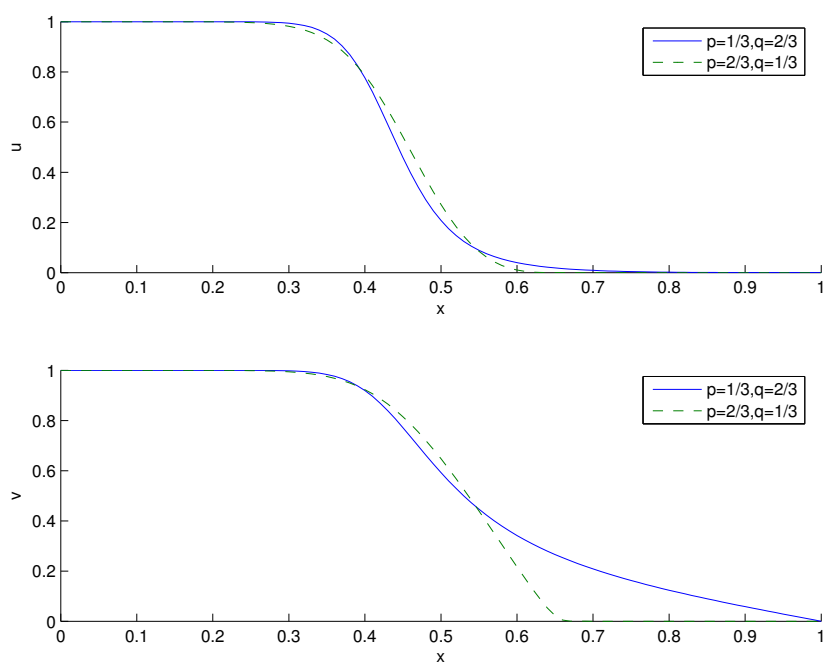

Figure 13. Solutions of the simplified Richards' model at $t=0.05$.
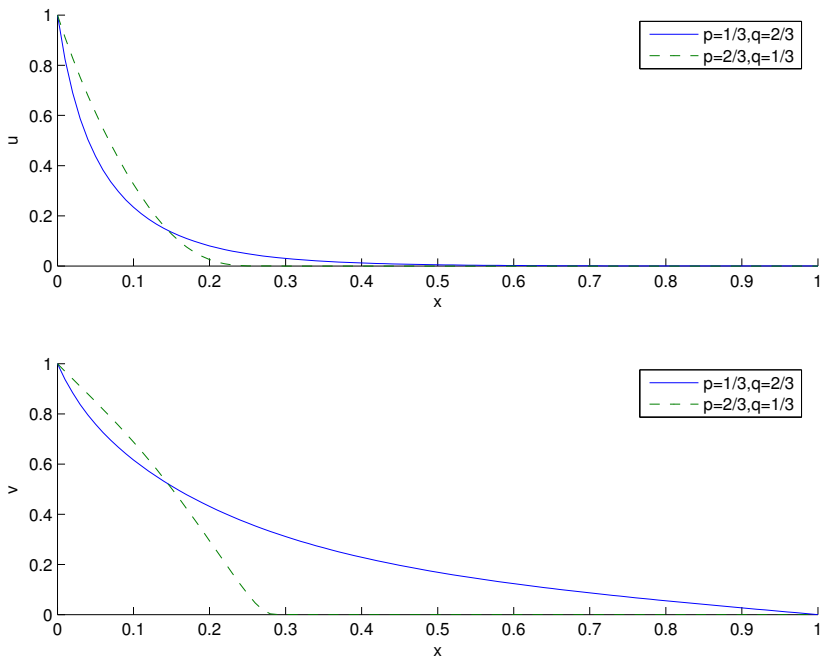

Figure 14. Solutions of the simplified Richards' model at $t=0.5$.
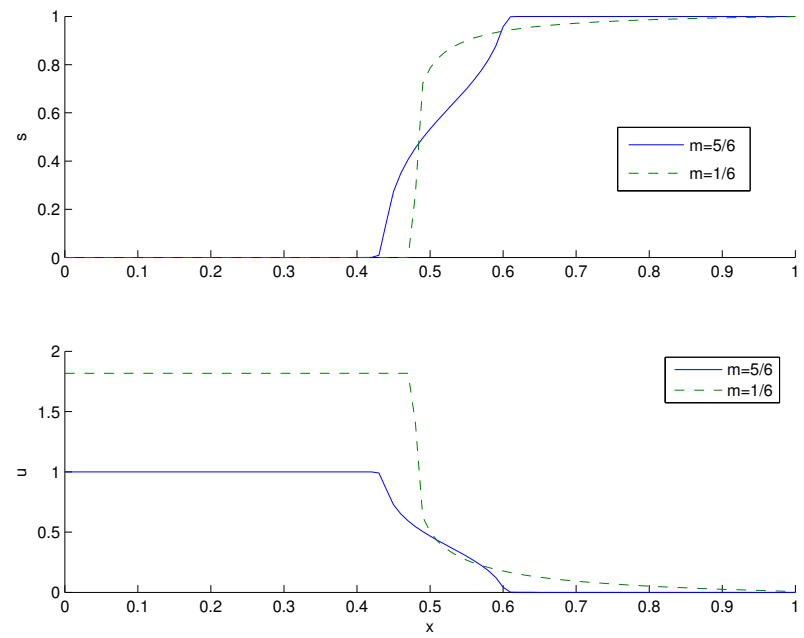

Figure 15. Solutions of the two-phase model for $m=$ $5 / 6$ and $m=1 / 6$ at $t=0.05$. The loss of finite speed of propagation for the $m=1 / 6$ case causes the entire domain to become unsaturated instantly.
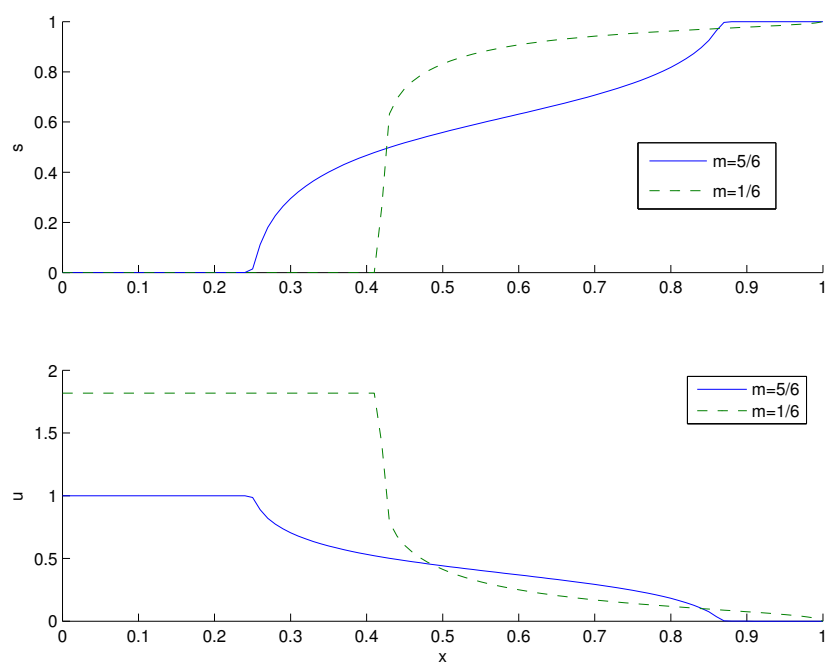

Figure 16. Solutions of the two-phase model for $m=$ $5 / 6$ and $m=1 / 6$ at $t=0.5$. 

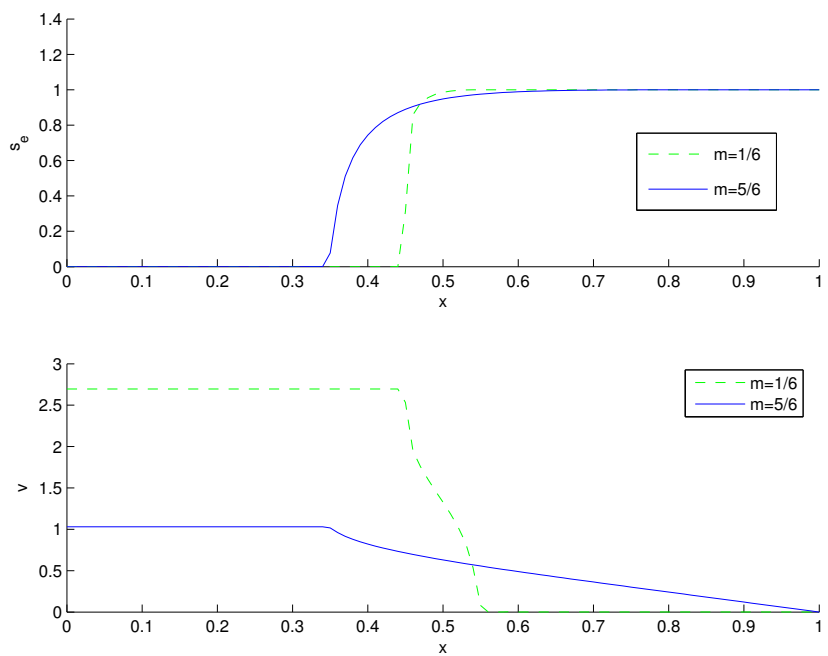

Figure 17. Solutions of Richards' equation for $m=5 / 6$ and $m=1 / 6$ at $t=0.05$. The loss of finite speed of propagation for the $m=5 / 6$ case causes the entire domain to become unsaturated instantly.
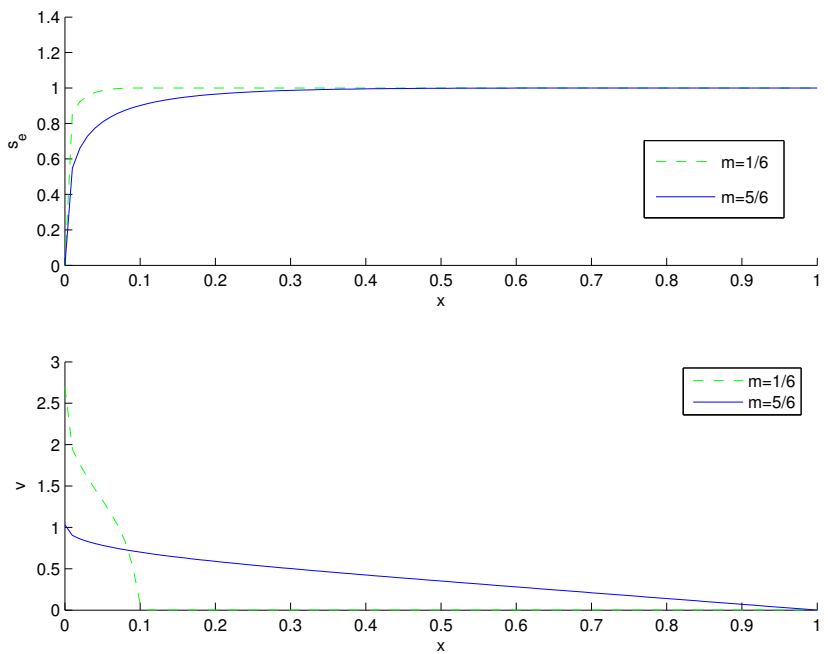

Figure 18. Solutions of Richards' equation for $m=5 / 6$ and $m=1 / 6$ at $t=0.5$. 